\title{
The implications of bile in the stomach
}

Possibly the most famous stomach in history, studied with pioneering persistence 135 years ago by Dr Beaumont, and belonging to Alexis St. Martin, gave information about bile in the normal stomach which is quite astonishingly accurate in principle today.

Dr William Beaumont commented: 'Bile is seldom found in the stomach except under peculiar circumstances . . . when the use of fat or oily food has been persevered with for some time there is generally the presence of bile in the gastric fluid ... irritation of the pyloric end of the stomach with the elastic tube or the bulb of the thermometer generally occasions a flow of bile into this organ ... with the exceptions I have mentioned, it [bile] is never found in the gastric cavity in a state of health.'

He had the fortune to study this cavity through a long-lasting gastrostomy, and the difference between his observations and those made since with the apparently more precise technique of a tube in the stomach, which report on the whole that bile does regurgitate into the stomach in perfectly healthy individuals, was pointed out by Braithwaite 1 in 1942, who attacked the validity of stomach juice so aspirated as far as bile is concerned. It is known that persistent vomiting in normal people often produces bile-stained fluid; and the nausea that most intubated patients feel, with afferents stimulated from the soft palate to the pylorus, might well in this way account for the bile entering the stomach where it might not otherwise do so.

Concerning the diseased stomach, there has been slowly uncovered in the past 20 years the pattern in basic terms, of bilious entry into the stomach. James and Pickering ${ }^{2}$ and Watkinson, ${ }^{3}$ recording bile in aspirated stomach juice overnight, noticed that it appeared more often in patients with gastric than duodenal ulcer. Siurala and Tawast ${ }^{4}$ in a more precise study of 366 patients, using gastroscopy, biopsy, and Gmelin testing of the juice, found bile in the stomach 'more general' in patients with atrophy, and a fasting juice of high $p \mathrm{H}$. Du Plessis, ${ }^{5}$ with possibly less definite evidence from gastrectomy specimens, took the argument further, incriminating bile as the cause of gastritis and its complications, tentatively including carcinoma of the stomach. Capper and his colleagues ${ }^{6}$ devised a test wherein a tube in the duodenum was used to force $30 \mathrm{ml}$ of barium into that organ; he found with screening that normal patients showed no leakage of barium past the pyloric ring, while the majority of patients with gastric ulcers and all with achlorhydria did so.

Bile has come under suspicion therefore as a cause of the various inflammatory and degenerative afflictions of the stomach, and some ingenious experiments have set out to test this as a plain cause-and-effect phenomenon. Possibly the most positive of these was carried out by Byers and Jordan ${ }^{7}$ in a series of experiments on dogs, wherein an isolated patch of fundic mucosa was implanted into the gallbladder; the result, after nine months, was no change in the stomach mucosa, but inflammation in the surrounding biliary lining. In the same year, Belowski, ${ }^{8}$ again using dogs, anastomosed 
the gallbladder fundus to the stomach body, tying off the common bile duct; this produced mild inflammatory and atrophic changes around the anastomosis in the stomach; these changes disappeared in five months. The biliary tree was again found to have suffered chronic inflammation. Lawson, ${ }^{9}$ also in dogs, after a variety of gastric resections, described a more severe gastric inflammation in the area around the anastomosis: than the earlier workers: this could be due to $(a)$ the higher concentrations of bile allowed automatically after resection of part of the stomach, and $(b)$ the fact that his observations were made after three months, whereas Belowski ${ }^{8}$ described the inflammation he saw resolving after five months.

Evidence from experiments on dogs, creating free backflow of bile into the stomach, or allowing bile to contact its isolated mucosa, have thus in general not pointed to this leakage as a direct cause of general mucosal atrophy of this organ which keeps the bad company of gastric ulcer, achlorhydria, and gastric cancer in man.

The much vexed question has been carried further in a recent study. ${ }^{10}$ Experimental pyloric incompetence in a series of 10 dogs was shown to have no general effect whatever on the secreting surface of the stomach, as measured by weekly maximal stimulation tests, and finally by histology in up to six months.

In over 600 patients for routine pentagastrin stimulation tests, the samples of stomach juice were inspected for obvious bile, and the bilirubin content was measured in 213 of these, consecutive and unselected. Any error from the presence of a nasogastric tube was standard for the whole series. The group included 33 with a maximal acid output less than 10 m-equiv/hour and 32 who had had gastric surgery, and therefore no working pyloric partition. The results were related to the maximal acid output. It is well known that the figure of around $10 \mathrm{~m}$-equiv $\mathrm{HCl}$ in the post-stimulation hour marks the watershed between normal and atrophic gastric mucosa. ${ }^{11,12}$

The findings were interesting and unexpected. The normal stomach contained obvious bile in one-third of the resting samples. This may be in part due to the presence of the nasogastric tube, though an experiment in five dogs with Gregory cannulae in their stomachs ${ }^{13}$ failed to show that such a tube causes bile to appear in the stomach.

Twenty mg bilirubin entered the normal stomach during the resting hour, was present in one-third of samples, and at an overall concentration averaging $0.3 \mathrm{mg} \%$. After pentagastrin, the amount doubled, to $40 \mathrm{mg}$ in half of the samples, and the average concentration fell to about $0.2 \mathrm{mg} \%$.

One hundred and thirty $\mathrm{mg}$ of bilirubin entered the stomach in patients without a pyloric partition in the resting hour and was present in $56 \%$ of samples at an average concentration of $1.0 \mathrm{mg} \%$. After pentagastrin the amount fell, as would be expected in a common chamber with the main direction of flow distally, to $100 \mathrm{mg} /$ hour present in $67 \%$ of samples at an average concentration of $0 \cdot 8 \%$.

Patients with gastric atrophy behaved differently: $40 \mathrm{mg}$ bilirubin entered the stomach at rest, appearing in $50 \%$ of samples at an average concentration of $0.9 \mathrm{mg} \%$. After pentagastrin, the amount of bilirubin entering the stomach fell to $25 \mathrm{mg} \%$, though it was present in $60 \%$ of samples at an average concentration of $0.25 \mathrm{mg} \%$.

These figures imply two tendencies straying from normal in people with gastric atrophy. First, the pyloric barrier, and this must be a complex of 
action of the ring itself and the muscle of the chambers on either side, is inefficient at rest. Second, there appears to be a weakening of the normal response to pentagastrin in terms of the retropulsion of bile into the stomach. This impression is reinforced by the fact that in this group alone did the maximum concentration of bilirubin reached in any one of the samples fall after pentagastrin, and this to some extent is an indicator of the force of this response: it did so by $40 \%$, while rising by $40 \%$ in normals, and $20 \%$ in postoperative cases.

The condition of gastric atrophy thus carries the stigmata of an atrophic mucosa; of abnormally large amounts of bile appearing in the stomach for longer periods than normal, together with a reversal of the normal increase in amount following stimulation with pentagastrin. The two latter make suspicious the state of the pyloric barrier at rest, and the muscular responses in the foregut to pentagastrin, and point to a failure, possibly a common one, in a basic response on the part of these muscles to stimulation.

Muscle strip perfusion tests from the antral, pyloric ring and duodenal muscle of dogs ${ }^{10}$ showed that here pentagastrin was a consistent and powerful stimulant to the normal activity of the antral pump ${ }^{14}$ but had no direct action on the isolated ring muscle, and no notable effect on the duodenum. Histamine failed to stimulate the antrum consistently, but infallibly contracted the ring, and showed a marked stimulant effect on the duodenal muscle.

Does histamine normally stimulate duodenal activity? This complex of degeneration known as 'gastric atrophy' must clearly be much more deeply explored for its root cause: an increased amount of bile in stomach juice is but one of its components, and not on present evidence the cause.

There is meantime a practical application of this knowledge. Oesophagitis caused by bile, and bile salts appear to be the irritant responsible, ${ }^{15}$ is a vicious disease, much more likely to inflict haemorrhage ${ }^{16,17}$ and stricture ${ }^{16,18,19}$ on that fragile organ than peptic assault. Patients with gastric atrophy are, with postoperative gastric patients, at much higher risk from such an inflammation than those with normal acid secretion, and their surgical management should therefore be considered more urgently.

G. J. COLE

\section{REFERENCES}

${ }^{1}$ Braithwaite, L. R. (1943). The role of bile in duodenal regurgitation. Brit. J. Surg., 31, 3-9.

'James, A. H., and Pickering, G. W. (1949). The role of gastric acidity in the pathogenesis of peptic ulcer. Clin. Sci., 8, 181-210.

'Watkinson, G. (1951). A study of the changes in $p \mathrm{H}$ of gastric contents in peptic ulcer using the 24-hr test meal. Gastroenterology, 18, 377-390.

'Siurala, M., and Tawast, M. (1956). Duodenal regurgitation and the state of the gastric mucosa. Acta med. scand., 153 (6), $451-458$.

'Du Plessis, D. J. (1962). Gastric mucosal changes after operations on the stomach. S. Afr. med. J., 36, 471-478.

"Capper, W. M., Airth, G. R., and Kilby, J. O. (1966). A test for pyloric regurgitation. Lancet, 2, 621-623.

'Byers, F. M., and Jordan, P. H. (1962). Effect of bile upon gastric mucosa. Proc. Soc. exp. Biol. (N.Y.), 110, 864-866.

'Belowski, H. (1962). Does the duodenal content exert a harmful effect on the gastric mucosa ? Gastroenterologia (Basel), 98, 233-238.

'Lawson, H. H. (1964). Effect of duodenal contents on the gastric mucosa under experimental conditions. Lancet, 1, 469-472.

${ }^{10} \mathrm{Cole}$, G. J. (1968). On the intrusion of bile into stomach and oesophagus. M.Ch. Thesis, Queen's University. Belfast.

${ }^{11}$ Christiansen, P. M., and Johansen, A. (1966). Single gastric biopsy in subjects with low acid secretion after maximal histamine stimulation. Scand. J. Gastroent, 1, 86-93.

${ }^{13}$ Bock, O. A. A., Richards, W. C. D., and Witts, L. J. (1963). The relationship between acid secretion after augmented histamine stimulation and the histology of the gastric mucosa. Gut, 4, 112-114.

${ }^{18}$ Cole, G. J. Unpublished data.

${ }^{14}$ Code, C. F. (1967). Lecture to British Society of Gastroenterology, Dublin. 
"Moffat, R. C., and Berkas, E. M. (1965). Bile esophagitis. Arch. Surg., 91, 963-966.

${ }^{10}$ Palmer, E. D. (1960). Subacute erosive esophagitis associated with achlorhydria. New Engl. J. Med., 262 (18), 927-929.

17Payne, W. S., Andersen, H. A., and Ellis, F. H. Jr. (1963). The treatment of short esophagus with esophagitis by gastric drainage procedures with and without vagotomy. Surg. Gynec. Obstet., 116, 523-528.

${ }^{19}$ Helsingen, N., Jr. (1961). Oesophagit is following total gastrectomy. Acta chir. scand., suppl. 273, 1-21.

${ }^{10}$ Benedict, E. B. (1960). Benign inflammatory postoperative esophageal stenosis. Gastroenterology, 39, $285-292$. 